\title{
"I just leaned on it!" Exploring Opportunistic Social Discovery of a Technologically Augmented Cushion
}

\author{
Beatrice Monastero \\ Department of Computer Science \\ Aalto University, Espoo, Finland \\ beatrice.monastero@aalto.fi
}

\author{
David McGookin \\ Department of Computer Science \\ Aalto University, Espoo, Finland \\ davidmcgookin@gmail.com
}

\author{
Tapio Takala \\ Department of Computer Science \\ Aalto University, Espoo, Finland \\ tapio.takala@aalto.fi
}
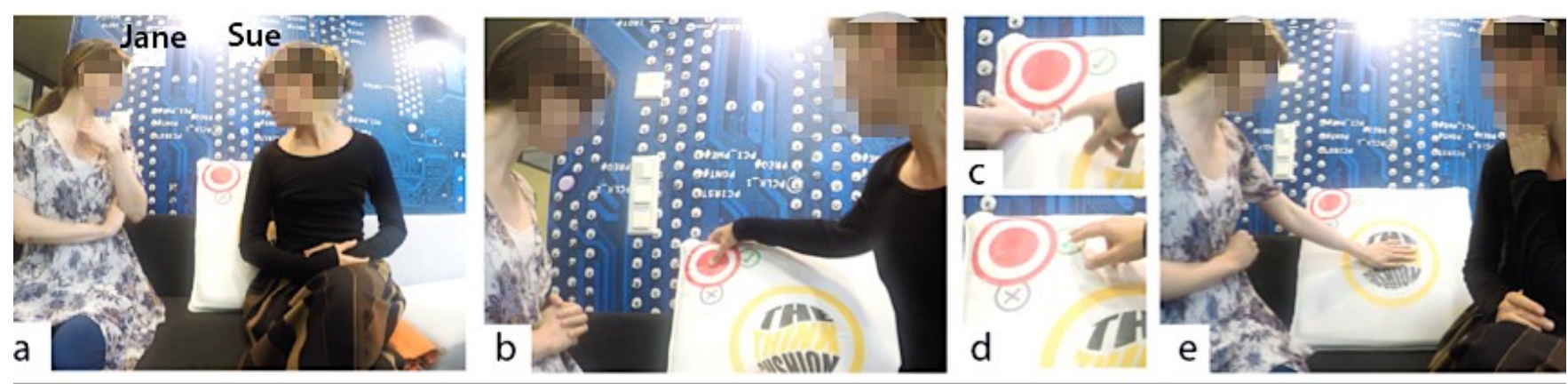

Figure 1. Example of interaction with ThinkCushion: a) Jane and Sue sit on the sofa, Sue leans on ThinkCushion triggering the playback of an audio message, b) The audio directs the girls' attention towards the cushion and they start exploring it. Sue presses an interactive area of the cushion that captures audio for 5 seconds signalling with blinking LEDs, c-d) after the recording two other areas of the cushion blink giving the option to save (green tick) or delete (grey cross) the audio just recorded. e) Jane presses the centre of the cushion to hear if the audio has been successfully saved or deleted.

\section{ABSTRACT}

While personal devices are often used to connect online with others far away, public media rarely offers opportunities to connect with collocated individuals. We explore novel interaction strategies to enhance opportunistic collocated sociality through technologically augmented daily objects. ThinkCushion is an augmented cushion allowing users to record and playback audio messages either explicitly or implicitly by leaning on it. We deployed ThinkCushion in an open coworking space and gathered quantitative and qualitative data over one month to unveil how individuals discovered it and interacted. We individuate three modes of discovery (serendipitous, spectated and facilitated) and their relations with situated socio-spatial aspects. We discuss the interplay of active and passive interaction modalities for locally accessing and creating content, and how verbal content can be used in either performative or informative ways. We suggest future research on how to design public technologies supporting collocated sociality already from the phase of technological discovery.

\section{Author Keywords}

Embedded systems; Opportunistic Interaction; Sociality.

Permission to make digital or hard copies of part or all of this work for personal or classroom use is granted without fee provided that copies are not made or distributed for profit or commercial advantage and that copies bear this notice and the full citation on the first page. Copyrights for third-party components of this work must be honored. For all other uses, contact the Owner/Author(s).

CHI '20, April 25-30, 2020, Honolulu, HI, USA

(C) 2020 Copyright is held by the owner/author(s).

ACM ISBN 978-1-4503-6708-0/20/04.

https://doi.org/10.1145/3313831.3376802

\section{CSS Concepts}

- Human-centered computing Human computer interaction (HCI); Haptic devices; User studies.

\section{INTRODUCTION}

We socially identify with others and form communities through engagement in shared activities and imagination, to construct "an image of the world that helps us understand how we belong or not" [55]. Wenger argues how tools for imagination such as language, pictures and TV shows, can increase individuals' awareness and curiosity about the presence and activities of others [55]. Enhancing curiosity $[26,38]$ and interaction $[9,34]$ between collocated individuals in public settings can also support wellbeing and generate social capital $[4,28,39]$. In coworking spaces social opportunities between collocated individuals can benefit cooperation by helping people to familiarize with each other and build trust [39]. However, this is usually supported mainly by organised, top-down activities (e.g. events, workshops), webpages or static posted material (e.g. posters and noticeboards) [54] that require users to be pre-motivated to access information and engage. Coffee breaks or the need to sit close to others (e.g. for limited space) can present opportunities for interaction. However individuals still need a strong motivation and social attitude to start conversation or even to recognise the value of connecting with others $[9,14,31]$. In coworking spaces private online social networks (SNSs), such as Facebook or Twitter, are the most used tools for social connection [23]. Yet SNSs are usually used to connect with distant others and may discourage connecting personally with individuals nearby [52], especially if their 
profile doesn't match personal expectations [23]. This often creates feelings of isolation and disconnection even when amongst others $[5,59]$. There is value in exploring how public technologies could be designed to support opportunistic social interactions between collocated individuals.

We explore a novel interaction strategy based on augmenting daily objects with interactive technologies capable of supporting situated sociality and the potential of locally generated verbal content. We test our hypothesis by augmenting a cushion (a daily object commonly used by a variety of people) with technology that allows users to create and access audio messages by pressing different areas explicitly or implicitly (by leaning on it). We introduced ThinkCushion for a month in an open coworking space that was seeking to support collocated interactions and colloquial atmosphere and analysed how individuals discovered it and interacted with it in relation to situated socio-spatial aspects.

\section{KEY CONTRIBUTIONS}

Beyond the specificity of our findings we contribute to future research on public social technologies by:

a) Defining modes of discovery through which inhabitants of public spaces opportunistically discover and learn interactions opportunistically (alongside daily activities and without instructions from the researcher).

b) Identifying relations between modes of discovery and situated socio-spatial aspects that future studies can consider and implement towards the creation of a framework for designing ad-hoc social discovery.

c) Presenting the benefit of novel mixed/multimodal interactions with embedded systems to support public opportunistic engagement with user-generated content.

\section{RELATED WORK. OPPORTUNISTIC DISCOVERY AND ENGAGEMENT WITH LOCAL PUBLIC CONTENT}

There are recognised challenges in purposing interaction with technologies to individuals in public settings. First, potential users need to notice the technology and its interactive qualities [22,32]. Then individuals need to be motivated to interrupt their current activity (e.g. even just walking with the aim to reach a specific destination $[35,47])$ to engage in interaction [53]. Researchers often recruit test users with flyers or instructions explaining how to use the technology $[25,58]$. There is a lack of understanding of how individuals could independently discover and interact with interactive technologies embedded in daily objects, without being motivated by the researcher or having considerable time to learn how to interact.

Discovery and learning of interaction are highly social and collaborative processes. Individuals can, for example, learn how to interact by observing other people interacting, e.g. through the honeypot effect [58]. However, this is mainly documented in novel group interactions using large public displays $[33,44,57,58]$. It is not clear how the honeypot effect may play out in more subtle interactions with augmented daily objects (such as furniture or living spaces). Furthermore, group interactions characterising the honeypot effect may not benefit less ludic spaces (e.g. coworking spaces) where individuals also work. Learning of interaction and appropriation also happen through collaborative and creative activities influenced by social context $[8,37]$, and often involve emergent patterns of collaboration and roles [50]. Quinones, for example, showed how experienced users could become technological facilitators and teach others how to use new software [42]. In this respect we hypothesise that technological facilitation by collocated people could support technological discovery and interactions in daily inhabited spaces where the researcher is not present to teach interaction. However, it is not clear how technological facilitation may arise in public interactions with daily augmented objects. Previous similar research has rarely considered the sociality of discovery, and particularly the relation between recurrent and new users, beyond the honeypot effect. These research gaps motivate our first research question:

\section{How do potential users discover augmented daily objects and learn how to use them (RQ1)?}

In tangible and embodied interaction research, a variety of common objects have been augmented with interactive technologies to affect users' behaviours in entirely reactive ways. Examples of this are floors delivering awareness of others while walking on them [35], benches changing shape to promote social encounters [27], tables synchronising the eating speed of diners by changing the distance of their plates [3], or illuminating to foster reflection around domestic activities [15]. However, most social interactions with public embedded technologies focus either on fostering face-to-face interaction [47] or accessing remotely generated content (and often turn into gaming activities). A minority of previous studies have illustrated how content generated locally by users has a high potential also for engaging curiosity and conveying contextually relevant social information [36,51]. However, it is difficult to motivate users to create public content as they often fear interacting in the "wrong" way, or lack a creative stance [25]. Nevertheless, users prefer accessing content that has been personally created rather than automatically retrieved from different sources [24,25,51]. Creating and sharing content from personal devices can partially solve issues of embarrassment due to public interaction $[2,30]$. However interaction through personal devices requires more effort from the users, who need to be motivated and prepare their devices to interact [21]. This can render local content access and creations more difficult to approach opportunistically alongside daily activities.

Lightweight implicit interaction modalities can allow, for example, to create social informational content through daily behaviours (such as walking [35]). However, it is still understudied how daily behaviours could be fostered to move users from implicit to active interaction and motivate them in 
creating content through ad-hoc interactions. This possibility is suggested by studies such as Bluetooth encounters [10], where users started changing personal Bluetooth name into more complex sentences addressing nearby people, to make it appear on a reactive monitor. Unfortunately, the study does not present how users discovered interaction and switched from implicit to active content creation. Golsteijn et al. [17], used a ringing phone on an interactive public wall to attract and elicit participants of a conference to lift the receiver and answer a survey. In this respect, auditory input could particularly suit on-the-go content creation by allowing a wide range of meaning to be potentially conveyed in a short message (e.g. users' identity, mood and context). These studies suggests how reactive signals and strangeness of the interface can attract the attention of passers-by and facilitate social reflection [9] and coordination [3]. Furthermore they exemplify how interaction metaphors transferred from familiar interaction contexts can ease learning of novel interactions [12].

We aim at gaining a better understanding of how public opportunistic interactions with informational content could be supported by leveraging daily actions with familiar objects augmented with unexpected interactive behaviours.

How can daily objects be augmented to motivate local creation and access of public verbal content (RQ2)? More specifically we aim at unveiling how the interplay of implicit and active interaction modalities can affect users' engagement with verbal content, and which kind of content would users record if not guided by questions/instructions.
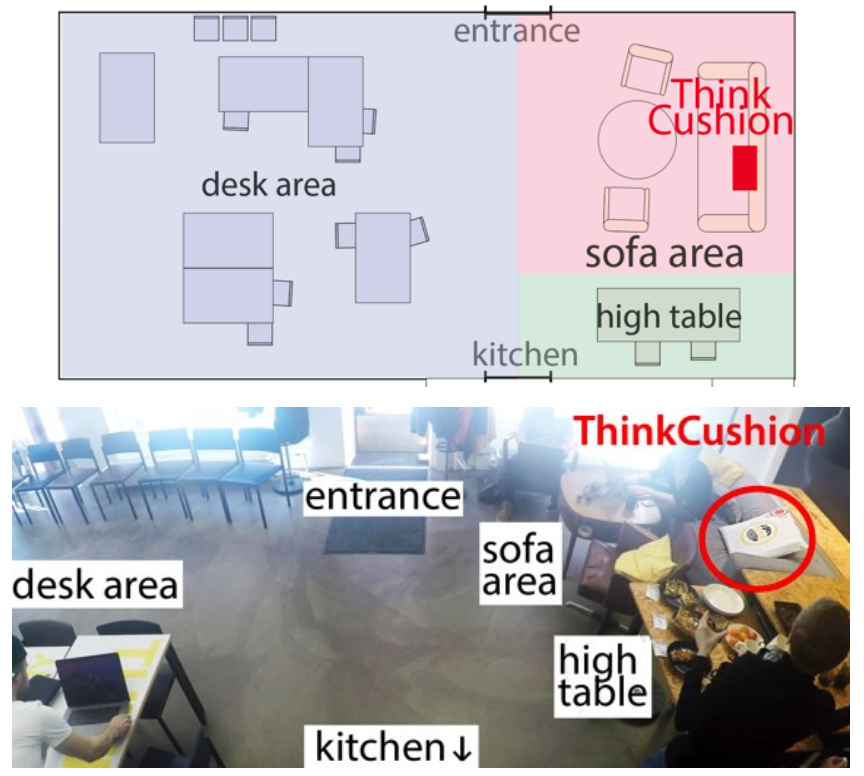

Figure 2. Map and picture of the Think Company's main room, showing the position of ThinkCushion in the sofa area.

\section{LOCATION: THE THINK COMPANY}

We investigate these issues in an open coworking space in Helsinki city centre, where individuals gather for both work and leisure, welcoming the possibility of interacting with others and joining proposed activities. Occupants of coworking spaces may have different roles (e.g. employees, visitors, event organizers) that can impact on how the technology is discovered and used. Furthermore, supporting sociality in coworking spaces is beneficial for the creation of trust and eventual collaboration $[2,28,39]$. We worked with the Think Company, an open coworking space and entrepreneurship society in Helsinki, Finland, for its specific spatial and social configuration. A friendly atmosphere of equality and inclusion distinguishes it from other more workoriented coworking spaces in the capital. However, interaction between occupants is facilitated mainly by programmed activities, such as networking events and workshops. When there are no such activities, employees need to envision strategies to preserve a colloquial atmosphere, to include people present in the area and avoid "library-like" silence and isolation. This is usually achieved by playing music on the speakers and welcoming discussion at medium/high voice level. Think Company's main room of ca. $25 \mathrm{~m}^{2}$ (Figure 2) is divided into a desk area with movable tables, a sofa area and a corner with a high table used mainly by employees to carry out computer work and organize refreshments during events. The open configuration of the space gives collocated individuals an overview of what happens in the space. A heterogeneous audience composed mainly of students and academics, entrepreneurs, researchers and visitors, visits the space for diverse reasons and at different frequencies.

\section{THINK CUSHION}

In order to address our research questions in the context of the Think Company, we chose to augment a daily object that individuals in the coworking space are likely to encounter during daily activities, especially in moments of relaxation, when they may be more curious towards others. ThinkCushion (Figure 3) is an interactive sofa cushion allowing users to record and playback audio messages by explicitly pressing different areas of it (active interaction) or by simply leaning on it (implicit interaction supported by the reactive feedback) (Figure 1). Individuals use cushions alongside daily activities in disparate ways: by moving, carrying, and passing them to other people. In order to facilitate our observations of how eventual group interactions and technological facilitations would arise, we positioned the cushion in the sofa area of the Think Company (as a variety of people sit on it and touch/move cushions while carrying out daily activities).

We iteratively developed ThinkCushion starting from a prestudy with non-technological prototypes in a similar social setting. Then we improved design ideas by paying regular visits at the Think Company before introducing the cushion, to familiarize with the space and situated practices. Bodystorming sessions [46] were carried out within our research group to envision interaction modalities and test components and their arrangement, in order to grant comfort in using the cushion both for interaction and for normal sitting/leaning. 
For the final design (Figure 3) we used a Raspberry PI 3B, a lightweight speaker, microphone and a battery pack. We designed ad-hoc circuit and soft switches from foam, tin foil and electrical shielding tape. Fast design cycles with informal usability tests were carried out in our department to test different interface design and ensure interaction affordances were clear. We fixed the final design (symbols and feedback) when all the functionalities become discoverable by pilot users in less than 2 minutes.

Users can trigger the playback of an audio message, randomly retrieved from a storage containing all saved messages, by touching the center of the cushion or leaning on it (Figure 1.a). In order to record a new message, users press the active area indicated by a red recording symbol (upper left in Figure 3), red LEDs will blink for 5 seconds indicating the time in which audio can be recorded (Figure 1.b). At the end of the recording time the audio is played back directly while giving the users the option of saving or deleting what they've recorded (Figure 1.c). Users save the message by pressing a green blinking tick area or delete it by pressing a grey blinking cross. If nothing is pressed the message is not saved for playback. If the message is saved it will play for the following 3 times when users activate playback, to give positive feedback about its saved state (Figure 1.d). From the third playback onward messages are randomly played back from the saved messages stored throughout the deployment history of ThinkCushion.

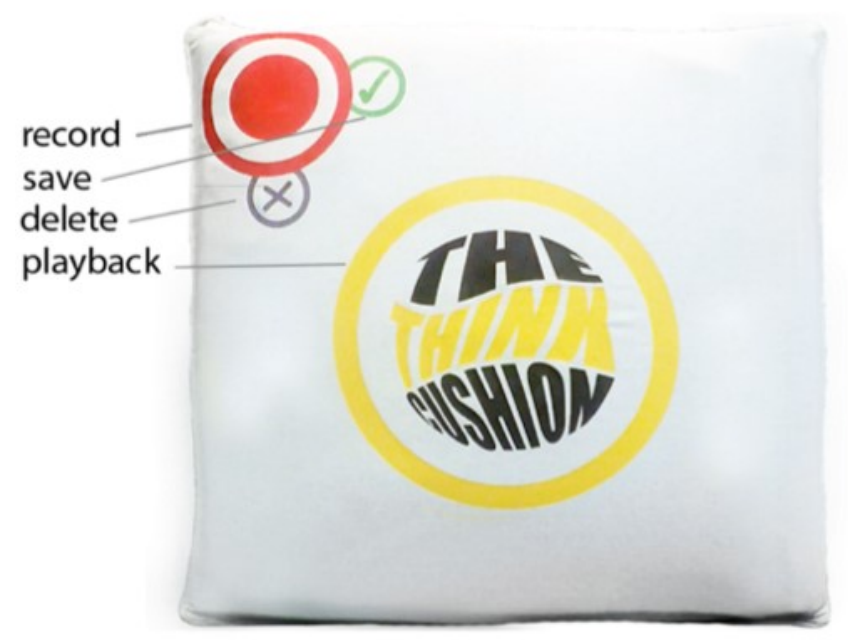

Figure 3. ThinkCushion interactive areas: record audio, save after recording, delete after recording, playback.

\section{STUDY OUTLINE}

For about one month a researcher was present for 3-4 days a week in the space to carry out preliminary interviews and conversations with employees and occupants of Think Company. This helped us to familiarize with situated inhabitants and practices and to envision ThinkCushion. We introduced the augmented cushion in the Think Company for a month during the standard opening hours (10am - 8pm) and collected both quantitative and qualitative data.

\section{Interaction Data Logs}

We logged each interaction instance (each time an interactive area was pressed) and stored the captured audio - saved, deleted and unselected messages. We coded and grouped the logged instances both by kind and temporal proximity to gain an overview of how participants interacted with ThinkCushion. The logged timestamp also served to indicate areas of interest for analysis in the video observations, and together with the captured audio supported validation of findings from the interviews.

\section{Video Observations}

In order to observe how situated activities affected the use of ThinkCushion, we carried out 1-2 hours video observations three times a week at times characterized by different activities (i.e. open working hours, during different events starting and ending, and across lunch breaks). Time-lapse videos ( 1 second intervals) were recorded through the length of the study. These allowed us to identify novel and recurrent users, the social unfolding of interaction around ThinkCushion and to support the validity of interviewees' statements. We classified how users interacted based on their level of awareness about the interaction, and its effect on collocated individuals. Notices were attached to the walls to describe what data was collected and to provide contact of the experimenters.

\section{Interviews and Surveys}

We invited occupants of the space to fill in a survey in situ or online (invited with fliers put on the desks) to unveil relations between technological discovery and individuals' frequency of visits, role in the space and proximity to ThinkCushion. Surveys and interviews were conducted in English. As ThinkCompany has a strongly multi-cultural set of visitors and entrepreneurs from nearby universities and startups, its "working" language is mainly English. A total of 31 participants, answered the survey. Of these 18 were females and 13 males, from 7 different nationalities with age ranging from 20 to 62 years old. Eleven of them, who visited the Think Company less than 3 times and never sat in the sofa area, answered not having noticed any particular technology. Thirteen over twenty users who mentioned having discovered the cushion also agreed to participate a further interview aiming at uncovering nuances of their interactions. Of these, 5 where recurrent users $(\mathrm{P} 4, \mathrm{P} 8, \mathrm{P} 9, \mathrm{P} 11, \mathrm{P} 12)$ that we interviewed twice to observe how their uses of ThinkCushion changed over time. We interviewed once the other $8(\mathrm{P} 1, \mathrm{P} 2$, P6, P7, P10, P13, P18, P20).

\section{Data Analysis}

We derived findings by triangulating between the different datasets through iterative phases of analysis and open coding $[6,13,43]$. We first analysed the data logs to individuate amount and duration of interactions over time, then we relied on the time stamps to spot areas of interest for analysis in the video observations. From analysis of the video observations 
alongside the data logs we were able to classify individual and group interactions and develop an initial set of codes based on our research questions and on users' awareness of interaction (aware, unaware). After analysis of the interviews and triangulation with the data already analysed, we implemented the initial codes to individuate specific modes of interaction. We finally added other codes to identify social interactions based on discussion, collaboration, and specific modes of discovery.

\section{RESULTS}

We present how users interacted with ThinkCushion, accessed and created content and perceived the value of interaction over time. We surface the nuances of how individuals discovered the technology through 3 main modes of discovery (serendipitous, spectated and facilitated) related to specific aspects of the interface and the socio-spatial organization of the Think Company.

\section{Observed Modes of Interaction}

We coded all interactions with ThinkCushion (for a total of 230 interactions), triangulating between data logs and video time-lapse observations. In $66 \%$ of these (152 interactions) users interacted with ThinkCushion when others were also present in the sofa area. We defined these "group interactions", based on the proximity between individuals. The remaining 34\% (78) were individual interactions by a user sitting alone in the sofa area. From a general perspective, the amount of interactions with ThinkCushion constantly varied irrespective of the presence of workshops and events in the area that might be expected to influence its use. Individuals often avoided interacting with it during workshops and events but were observed interacting during breaks. In this respect, the audio playback from ThinkCushion was often used to gather nearby individuals' attention to propose a shared break from work.

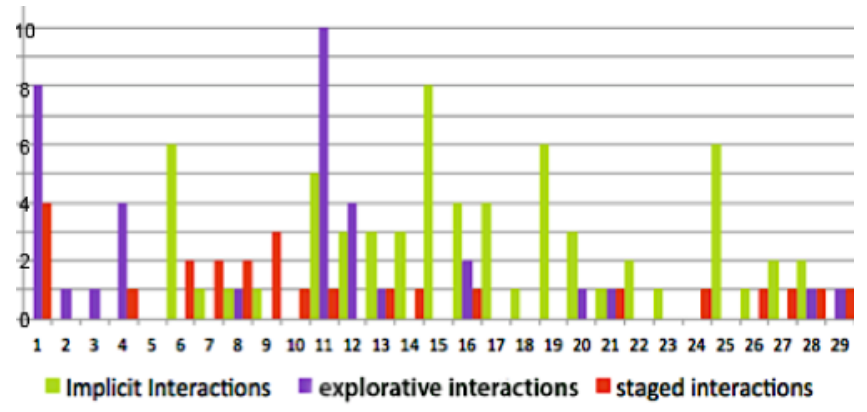

Figure 4. Implicit, explorative and staged interactions over time with vertical axis indicating the amount of interactions and horizontal axis the days of deployment of ThinkCushion.

Brief interruptions from on-going activities/work caused by the unexpected audio playback were not taken negatively by users, but they rather triggered brief connective reactions with others such as smiles and glimpses:

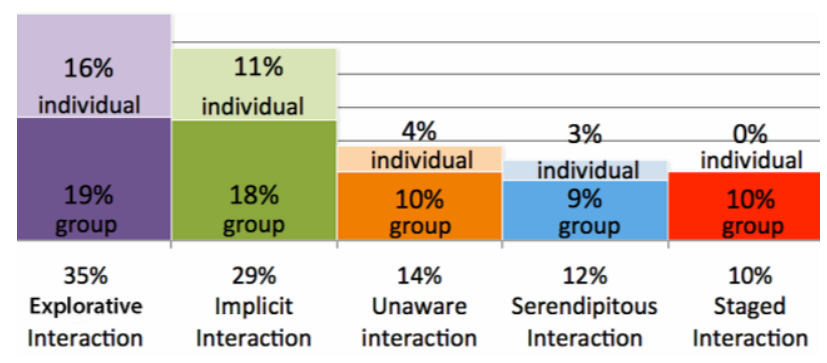

Figure 5. Percentages of the modes of 230 interactions, coded from analysis of interaction logs and video observations.

'At that moment, I was just doing something on my own and then I was like, "Oh, sound", and then someone was like, "Oh"[...] we were all busy working' (P7). A long-time employee of the Think Company explained how employees often had to prevent silent "library-like" situations by playing music and speaking aloud to invite others to be more colloquial and social. Interactions with ThinkCushion were also easily avoided by physically moving it away, either preventively by familiar users, or by new users after first interactions.

We identified five modes of interaction and classified them based on users' observable behaviors and awareness of interaction:

- Explorative 35\%: users interact consciously with ThinkCushion through ad-hoc behaviors to explore how it works or to discover what others recorded.

- Implicit 29\%: users interact consciously merging interaction with normative use of cushions without interrupting their daily activities.

- Unaware 14\%: users inadvertently activate playback but don't show awareness/attention about it, e.g. during loud parties masking the audio.

- Serendipitous 12\%: users become aware of Think Cushion's interactive behavior by touching or leaning on it (passing from unaware to explorative interaction).

- Staged 10\%: users interact by playing back or recording messages with exuberant behaviors with the aim of triggering reactions from nearby listeners.

Explorative interactions mainly happened at the start of the study when ThinkCushion was still a novelty and all users were exploring how it worked (more often in the first week, rarely after the second week of deployment, as highlighted in Figure 4). Recurrent users soon developed ways to use ThinkCushion partly as a prop to trigger reactions from collocated individuals in staged interactions. They recorded and accessed content mainly for performative aims, to trigger reactions (e.g. eye contact, questions, and smiles) from people nearby hearing the direct playback or triggering playback of saved messages subsequently.

For this reason, content such as sounds, laughs, songs, was recorded ( $90 \%$ of messages), rather than more structured information. Figure 6 presents an example of how collocated users accessed content to stage interaction aimed to trigger 
nearby people's reactions or gather their attention and proposing a shared work-break: 'Today was funny when we were sitting and A. sat next to me and leaned and pushed the cushion and she was like 'Oh, what's happening'? But it happened already before for her she already knew about it' (P11). The performative use of the audio recording functionality to trigger reactions from situated people, both in the moment of creation and in follow-up access, was explained as: '[P8] was carrying it around and recording people's voices on it and then he was repeating them and laughing' (P6).

Implicit interactions emerged from the $6^{\text {th }}$ day of deployment of ThinkCushion (Figure 4) showing how recurrent users understood and appropriated ThinkCushion quickly, being able to access messages simply by leaning on it without interrupting other activities: 'I learned that you can play the previous recordings, then I was excited about that. Then, at some point I forgot about it or I used it as a part of the area. For example, when I was writing my thesis and I was leaning, it was nice to hear something else than my own thoughts every now and then' (P12). However, while recurrent users continued to access content over time through implicit interactions, these did not afford recording new content. The lack of updates and the performative use of content could not support lasting value of interactions: 'I use it just as a sitting cushion but after a while I move it away [...] I would like if it would add more value to work, still people choose to record noises...or maybe this is what is needed in the space, balancing serious work with relaxing fun' (P8). On the one hand staged interactions had positive social effects insofar as they were acted to cause connective reactions between collocated individuals. Alternatively, the possibility of both recording and accessing meaningful content in implicit ways would have granted more value in long-term recurrent interactions, but raise content-publishing issues, as users need to actively agree to record a public available message.

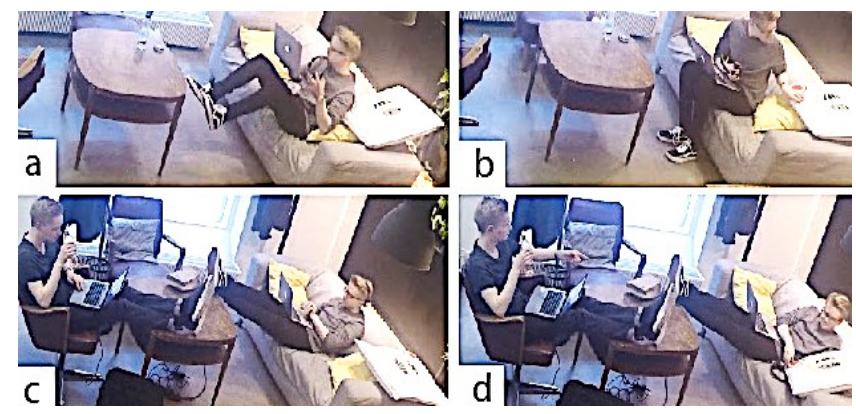

Figure 6. Example of staged interaction to synchronise workbreak: a) a user discovers the cushion serendipitously; b) he explores its functionalities c) a friend joins the sofa area to work e) the initial user interacts with ThinkCushion to catch his friend's attention and to invite him for a work-break.

Modes of Discovery and Socio-Spatial Relations

We identify and define three modes of discovery: a) serendipitous, b) spectated and c) facilitated. Quotations from interviews help presenting how modes of discovery related to specific social and spatial aspects of the deployment location.

\section{Serendipitous Discovery}

As outlined in Figure 5, 12\% of interactions referred to episodes of serendipitous interaction, in which new users discovered the cushion and interact with it by accidentally leaning on it and triggering audio playback. Five of the 13 interviewed participants (P1, P7, P9, P18 and P20) mentioned having discovered ThinkCushion serendipitously and provided insights on how this happened.

Serendipitous discovery happened when potential users sat on the sofa and triggered audio playback by leaning on ThinkCushion. The initial confusion was then quickly solved by recognizing familiar symbols suggesting the interactive aspects of ThinkCushion: 'I sat on the sofa and I leaned on it, and I was like, "Oh, sound"...it was confusing at first because I didn't know where the sound came from, but I figured it out pretty quickly. I could hear it was coming from behind me, and I was like, "Okay, oh, it must be the pillow", because there were also symbols on it that made me understand that it was for recording, it was clearly labelled' (P7). Also it was described how the interactive behaviour of ThinkCushion, while being unexpected and unordinary, could also be easily understood by users with previous experiences with similar interfaces: 'I leaned my back on it, and it started talking [...] I thought of the toys that I've had when I was small [...] so that is why I first thought that the pillow works in the same way'(P9). When users sat in a group in the sofa area, serendipitous discovery in most cases (16 out of 21) lead to collaborative exploration through discussion and group interaction. The surprise effect, caused by an unexpected audio, triggered discussion with nearby individuals and collaboration in exploring ThinkCushion: 'we started wondering what is it about, is it a recorder or something? And then my other colleague and I started pushing those buttons' (P1). While it is not possible to identify from the video observations the level of familiarity between individuals sitting together in the sofa area, interviewees described how discussions and collaboration were also triggered between nearby strangers: 'I was talking to $P$ and then I heard the sound from behind me, and $P$ was looking at me like, "What did you get scared for?" "I think this pillow is talking". And then I tried to ask what the pillow is from the other person who I didn't know, and she said that it records as well [...] so $P$ wanted to make a new recording [...] and then $M$ was playing' (P20).

These examples describe how the transition from discovery to first interaction was sustained by the interplay of the surprise effect (generated by the unexpected audio coming from the cushion) followed by recognition of familiar features in the cushion interface and behaviour. For most the unexpected playback was welcomed, considered fitting the colloquial atmosphere of the Think Company and the surprise effect generated caused brief connective reactions 
and social interactions between collocated people (eye contact, discussions and collaborative explorations).

\section{Spectated Discovery}

Four of the 13 interviewed participants (P10, P11, P12, P13) first interacted with ThinkCushion in explorative ways and explained having discovered it by seeing others interacting. We define this "spectated discovery". Some participants only discovered the playback of recorded messages at first: ' $I$ noticed that there was some workshop thing going on and the space was pretty quiet. Someone was working there, and they made some loud noise'(P13). Others had a wider understanding of how ThinkCushion works conveyed by spectating more complex interactions: 'you can record something and then you can play it again if someone touches it [...] I just saw what other people were doing and then caught that' (P10). In spectated discovery the actor may be unaware of serving as learning example.

Staging aspects such as positioning and size, appearance and the presence of contextual cues, attracted potential users' attention yet not directly leading to discovery of interaction. Environmental masking (i.e. noise, people and objects obstructing the view), distance from ThinkCushion, and strength of attention allocation on other tasks could drastically affect the visibility necessary for spectated discovery: 'I haven't paid that much attention because I've been busy doing my stuff. [I did] When I sat on the sofa and, obviously, people were very close by, otherwise, if I'm on the other side of the room I wouldn't pay much attention. [once] I was sitting there and there was a presentation going on and I pressed it by accident [...] but nobody minded that much' (P11).

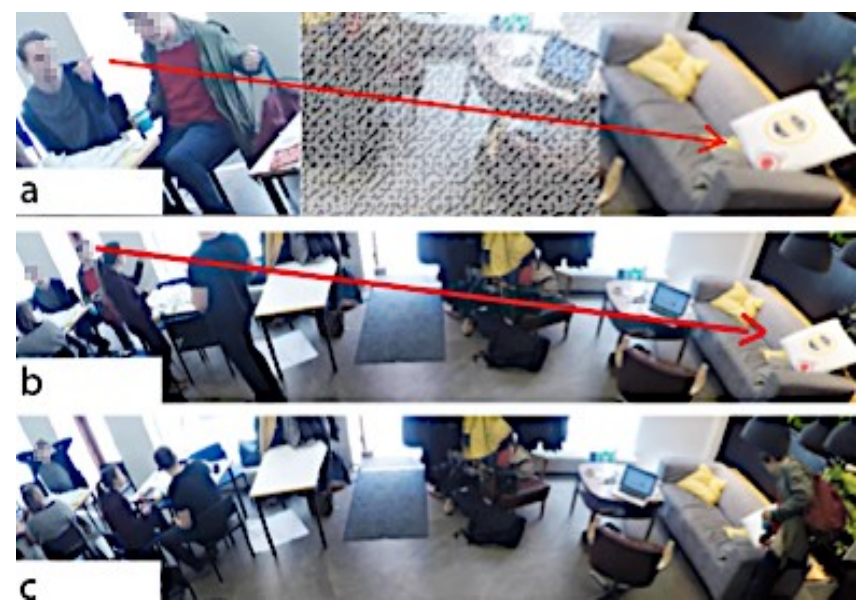

Figure 7. Example of informed facilitation: a) a user familiar with ThinkCushion (technological facilitator) points it out to a bystander during a workshop; b) the bystander notices ThinkCushion and c) interacts with it after the workshop

The magnitude of interaction (i.e. how loudly and visibly someone interacts) could also render interactions more obvious and sustain spectators' curiosity for a time long enough to learn interaction. Magnitude of interaction can also relate to unfamiliarity and volume of performed behaviours, audio feedback, completeness and clarity of interactions carried out: 'I saw when $J$ was here and he recording something and then pressed the button. He was laughing and then the pillow was laughing as well so it was like, "Oh okay, that's how it works"' (P12).

Frequency of interactions must also be considered, as individuals may occupy the stage for a long time without interacting (e.g. while carrying out a task on the sofa area), thus preventing possibilities for spectated discovery.

\section{Facilitated Discovery}

The remaining 4 of the 13 interviewed participants (P2, P6, P17, P8) mentioned (or were videoed) discovering ThinkCushion suggested by others already familiar with it.

A participant describes how a host introduced ThinkCushion to her, inviting her to interact with it and collaborated in the discovery of its functionalities: 'I think it was one of the hosts that introduced me to the pillow, [...] she was asking: "Have you already met our new cushion?" And I had no idea what it was, but R just gave it to me and said that I should figure it out or try it out, and then I pushed the middle of the button and it said something [...] then she pushed the record button, and then I blubbered something, and then it came again' (P2). We call it informed facilitation when an accustomed user introduces the technology to others through explanation, rather than by engaging in practical demonstrations. Figure 7 shows an asynchronous example observed from the time-lapses.

We can distinguish performed facilitation when users already familiar with the technology introduce others to it by performing with it to demonstrate functionalities (e.g. in staged interactions): 'the other board members started to play with it being aware of me [...] Then pointing it out to me' (P17), and 'I told one of my friends "check this out" and pressed the button to record a message [...] and they interacted the same way' (P7).

In other cases, technological facilitators introduced ThinkCushion to new users who asked about it after having recognized it as a new element in the space. We call these instances induced facilitation. In these cases, potential users may individuate the object alone (in serendipitous ways), but it is the intervention of a technological facilitator motivating them to interact: 'People are asking what is it, just in general, actually I encourage them to press it' (P12).

Fear of ruining the validity of the study and missed feeling of ownership, due to, for example, knowing the cushion belongs to a research project, can prevent familiar users from becoming facilitators. Individuals with official roles as social animators, more familiar with the space and the technology or social by attitude, easier assumed the role of technological facilitators. Four of the 8 interviewed hosts mentioned or were noticed introducing others to the cushion verbally (P13) or through demonstrations (P8, P9, P11). 
A social character introduced both colleagues and general visitors to ThinkCushion as a way to support conversations or their mood: 'I've been showing it to my friends and all sort of people, people gets in good mood because of the cushion, so that is good' (P8). Nevertheless, feeling of ownership and role as social facilitator were not the only factors motivating users to become technological facilitators. New users where noticed introducing ThinkCushion to others (especially friends) motivated by the enthusiasm of the discovery: 'In a few moments you figure out how the cushion works and it is funny so I wanted to introduce it to my friends who came in later' (P2).

Situational fit affected technological facilitation. For example, users were noticed waiting for the right moment to introduce ThinkCushion to others as a way to propose a shared work break. In Figure 8 a user who discovered ThinkCushion serendipitously by accidentally triggering playback during a workshop, and moved it away, was then found using it as a conversation trigger later on.

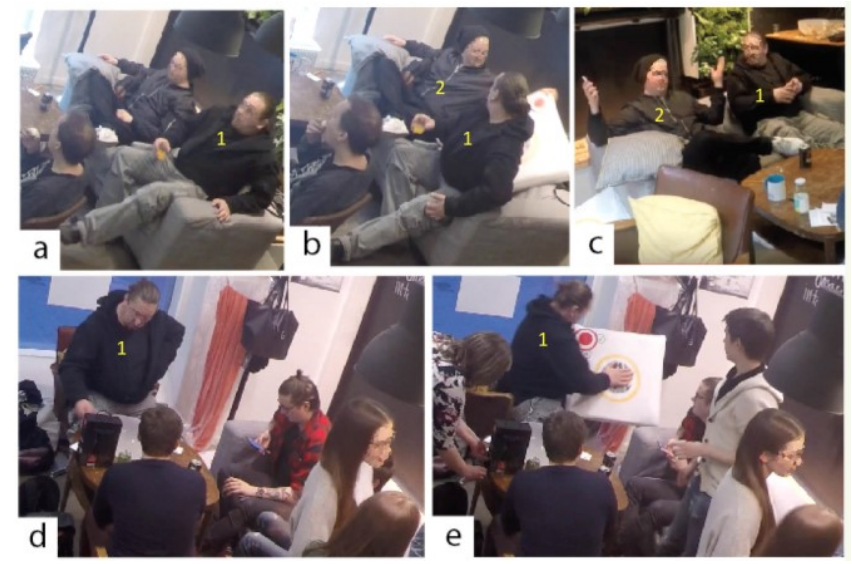

Figure 8. Performed facilitation for situational fit: a) user (1) discovers ThinkCushion during a workshop; b/c) he asks a nearby unacquainted (2) about it; d/e) after the workshop user (1) introduces the cushion to unacquainted workshop participants as a way the break the ice for conversation.

\section{DISCUSSION}

We present how the individuated modes of discovery and related contextual relations extend previous work on users' first engagement with public technologies. Then we draw a comparison of how differently augmented daily objects (e.g. silent or graphic interfaces, verbal or abstract content) can be used to support different forms of public interaction with user generated content. We suggest how daily objects could be designed to leverage and enhance opportunistic social interaction between collocated individuals already from the phase of discovery.

\section{Considering Modes of Discovery and Contextual Relations to Leverage and Enhance Situated Sociality}

Research in digital media art has shown how users engage with interactive technologies, transitioning from bystanders (unaware of the existence of the performance) to witting spectators, then participants (exploring interaction) and actors (knowledgeable users) [20,48]. We contribute with an understanding of how bystanders can gain awareness of the performance frame without being pre-informed by the researcher, and opportunistically engage in explorative interaction. We have individuated 3 modes of discovery (serendipitous, facilitated and spectated) and their relations to socio-spatial aspects of the deployment location (RQ1).

Previous research has documented strategies for drawing observers into active interaction by optimising visibility of other individuals interacting [21,58]. By augmenting a daily object with reactive feedback we aimed at testing a novel interaction strategy that supports opportunistic discovery of embedded systems also in single users' interactions, when it is not possible to observe others interacting. It has already been noticed how normative use of familiar objects can be defamiliarised to modify, guide or empower people's daily actions $[3,35]$. We extend this research by exploring how defamiliarising a daily object through reactive feedback and extraordinary behaviours supported serendipitous discovery and users' transition to explorative interaction also in singleuser interactions alongside daily activities.

In serendipitous discovery unwitting bystanders (to use performance metaphors $[20,48]$ ) discovered the augmented object while normally behaving with it (e.g. triggering audio playback from ThinkCushion by leaning/touching it). Theories of embodied cognition also noted how individuals easily and quickly adapt to unexpected situations, so that technologies can be designed to cause disruptions of normal behaviors to attract attention and facilitate reflection $[19,40]$. We could in fact observe how, even if unexpected reactive feedback could cause some disorientation at first, users swiftly become curious and engaged in exploration. Bystanders transitioned from discovery to engagement by first easily locating the signal source (proving the benefit of co-located over remote feedback [41]) and then guessing interaction thanks to the presence of familiar symbols that made interaction affordances visible at-glance.

The brief interruption of daily activity, caused by the reactive audio feedback, was not perceived as disturbing, but was rather welcomed as an opportunity to socially connect with collocated others. Users could choose to explore interaction or easily drop out by moving the cushion away or just avoid touching it. While the sociable atmosphere of the deployment location helped this consideration, further studies are needed to prove the benefit of this interaction strategy in locations characterised by different levels of sociality and familiarity between collocated individuals.

We invite future research to further study how serendipitous discovery and at-glance visibility of complex explicit interactions (e.g. publishing content) can be supported by the interplay of:

- Surprise effect (e.g. reactive feedback from daily objects)

- Cultural familiarity (normative uses and familiar symbols)

- Easy drop out from interaction (e.g. movable interfaces) 


\section{Facilitated Discovery}

Previous research on collaborative work software [42] has documented how users become technological facilitators when highly knowledgeable on how to use a technology. We observed how users willingly introduced the technology to others either verbally (informed facilitation), by acting with it (performed facilitation) or by answering potential users' questions (induced facilitation), also motivated by:

- Discovery satisfaction, felt after discovering the augmented object and independently learning to use it through fast trial and error process.

- Feeling of ownership, e.g. space occupants more familiar with the space may feel more agency in introducing others to the technology while sporadic visitors may fear to be using someone else's belonging.

- Situational fit, The ambiguous role of ThinkCushion allowed individuals to adapt its use to fit different situations, such as supporting, opening and closing conversations, synchronizing work breaks, triggering social reactions, facilitating in these ways others' discovery.

- Users' character and role. While social animators or extroverted individuals may easier introduce the technology to others, we also noticed more sporadic or introverted visitors becoming technological facilitators by occasionally using ThinkCushion as an icebreaker and ticket to talk $[45,56]$.

We suggest future research should deepen the study of how users may be motivated in becoming technological facilitators by design aspects such as fast progressive discoverability (supporting discovery satisfaction), branding/labelling (explaining ownership), and open function of use (for situational fit). In order to study the working of facilitation in public settings, we also emphasize the need of extending the analysis of interaction further from the area of direct interaction with the interface [7,11]. A methodological suggestion is relying on data gathering methods such as spatially and timely extended video observations, surveys/interviews, logs and triangulation between the datasets.

\section{Spectated Discovery}

Previous work noticed how visibility of a novel technology and engagement is facilitated by the honeypot effect [41] and specific spatial organisations [11]. In spectated discovery we noticed collocated potential users discovering interaction by observing others interacting also without the need of honeypot effect. Our findings are relevant especially for design of spectated discovery in locations where group interactions may disrupt daily practices (e.g. coworking places, libraries, cafés). Taking ThinkCompany as example, we noticed that users often moved ThinkCushion and reconfigured the space to facilitate specific activities (e.g. speeches, group work, events, open working hours). So it is necessary to extend theories of engagement based mainly on spatial configurations [11] to better account for more spatially dynamic responsive environments [1].

We propose extending the term "spatial organisation" to consider the dynamic relation between structuring aspects of the location [11], proxemics (e.g. distance of collocated users from each other and the interaction stage)[18], positioning and movability of the technology. Furthermore we highlight how areas of activation, interaction gaps [11] are easily reconfigured by design aspects of the interface and situational qualities of interactions.

We propose the term magnitude of interaction to comprehend aspects such as intensity/strangeness of the feedback, exuberance and number of users, affecting visibility of interaction. For example, the audio feedback of ThinkCushion could be more or less intense due to different amplitude levels of recorded messages, or noise levels in the room. Users could interact in more implicit ways, requiring less attention from individuals located further or could choose stage interactions to provoke social reactions.

Furthermore, we bring into consideration how frequency of interaction (how often someone interact) can strongly affect the chances of spectated discovery. In our interaction case, for example, a group of users could occupy the space of potential interaction close to ThinkCushion for a long time without interacting (e.g. while carrying out work), shielding access and visibility to others [21] .

We invite future studies to further develop these sensitizing concepts towards the formation of a framework for opportunistic social discovery. We aim at designing for different modes of discovery attuned with the specific interaction contexts. It is not clear for example how degree of strangeness of the interface may affect at-glance understanding of more complex explicit interaction (e.g. recording content) after serendipitous discovery. While familiar symbols can help at-glance guess of unexpected interactions, a too clear mapping may also lower discovery satisfaction and novel users' motivation to inform others (facilitated discovery). More or less visible or frequent interactions (magnitude and frequency) could be noticed in a specific distance range. This could be designed, for example, to favour facilitated over spectated discovery in environments where sociality should be supported but high magnitude interactions may disturb (e.g. in a library). More ludic environments such as parks or public squares may instead benefit from higher magnitude interactions attracting groups of users in honeypot effect.

\section{From Implicit to Opportunistic Active Engagement with User-generated Verbal Content}

Previous research has identified the challenges in publicly engaging users with content creation, even when motivated by the researchers (e.g. due to lack of time and creative/ social inhibition [53]). Research in embodied interaction also noticed how embedding technology within the already ongoing set of activities can facilitate individuals' 
engagement meanwhile accomplishing what they were already doing [27]. However this theory has been tested mainly by augmenting daily objects in 'silent' ways (e.g. solely reactive or not affording engagement with usergenerated semantic content [3,27]). We empirically tested augmenting daily objects as a way to support opportunistic access and creation of informational social content. We used a cushion that can commonly have graphics on it, to study how the interplay of silent/physical, graphical and sociospatial cues could guide users from implicit to active engagement with verbal content.

From an embodied perspective, normative ways of using the object [3], behaving in the space [16,29], the social atmosphere and users' familiarity with interface symbols, all contributed in facilitating users' transition from implicit to active interaction. Making access to interaction implicit to daily actions served not only for serendipitous discovery, but also remind users about the possibility to interact (opportunistic engagement). However we notice that messages where recorded mainly for performative rather than informative reasons (e.g. as a "ticket to talk"[45] with others, synchronize work breaks and trigger connective reactions such as smiles, eye contact, comments). As content was free (there was no guidance from the researcher on what to record, differently e.g. from Voxbox [17]), it is not clear what influenced users towards performative rather than informative uses. Would for example positioning ThinkCushion where individuals sit alone, motivate them to record information for the person who will sit next, rather than performing staged interactions for nearby individuals? Would the presence of informative messages motivate users to record similar content (e.g. for social imitation [49])?

We also noticed that recurrent users mainly interacted with ThinkCushion in implicit ways over time, causing less frequent content updates and losing interest for the informational content of messages. Previous studies succeeded in delivering social information solely with abstract representations generated by mimicking users' daily behaviors (e.g. [35]). However, implicit content generation would raise ethical issues with verbal content, as users need to give permission to record and publish. We encourage future studies to critically consider how different degrees of content abstraction/anonymity and mixed modes of interaction (e.g. implicit/active, verbal/tangible) may either support informative or performative content uses. We aim at balancing performative and informative behaviours to enhance different aspects of sociality from social imagination, awareness, and face-to-face interactions.

\section{LIMITATIONS AND FUTURE WORK}

We noticed how in the Think Company content was created mainly as a way to perform interactions, however it is not clear how content use may vary in different deployment locations (e.g. less colloquial/social environments). A limitation of our experiment is that it only covers a particular design with limited interaction modalities serving as a case study answering our questions in that context. In this respect the socio-spatial aspects of the Think Company allowed us to gather a rich set of findings about the dynamics of discovery and the use of ThinkCushion as an augmented daily object. Future studies will consider deployment in different locations to deepen the understanding of the relations between discovery and use of augmented daily objects and different socio-spatial aspects.

\section{CONCLUSION}

We have presented how daily objects could be augmented with interactive behaviours to support opportunistic technological discovery and social interactions between collocated individuals alongside daily activities. We highlight the potential for implicit interaction and reactive feedback from daily augmented objects to ease users' transition from opportunistic discovery to exploration of novel interfaces. We have defined three main modes of discovery (serendipitous, facilitated and spectated) and how these both leverage and affect situated sociality. In this respect we documented the role of surprise effect, cultural familiarity and easy drop out in serendipitous discovery, we highlighted the effects of discovery satisfaction, feeling of ownership, situational fit and users' character and role in motivating technological facilitation. We implemented sensitising concepts addressing how dynamics of spatial organisation, interaction magnitude and frequency can affect spectated discovery. Finally, we individuated how users can engage with public verbal content weather for performative and informative reasons, and their value in social interaction over time. We encourage future studies to draw on top of the individuated relations towards the creation of a framework for opportunistic social discovery and interaction in daily augmented spaces.

\section{REFERENCES}

[1] Ian Bently, Alan Alcock, Paul Murrain, Sue Mcglynn, and Graham Smith. 1985. Responsive environments. Architectural Press, Elsevier.

[2] Mark Bilandzic, Ronald Schroeter, and Marcus Foth. 2013. Gelatine: making coworking places gel for better collaboration and social learning. Proceedings of the 25th Australian Computer-Human Interaction Conference ( $\mathrm{Oz} \mathrm{CHI}$ '13), ACM, 427-436. http://doi.org/10.1145/2541016.2541027

[3] Laurens Boer, Robb Mitchell, Agnese Caglio, and Andrés Lucero. 2015. Embodied technology: Unraveling bodily action with normative types. Human Factors in Computing Systems (CHI'15), 1711-1716. http://doi.org/10.1145/2702613.2732899

[4] David Bradley, Jane Bradley, Mike Coombes, and Emmanouil Tranos. 2009. Sense of Place and Social Capital and the Historic Built Environment.

[5] Scott Caplan, Dmitri Williams, and Nick Yee. 2009. Problematic Internet use and psychosocial well-being 
among MMO players. Computers in Human Behavior 25, 6: 1312-1319. http://doi.org/10.1016/j.chb.2009.06.006

[6] Juliet Corbin and Anselm Strauss. 2008. Basics of Qualitative Research (3rd ed.): Techniques and Procedures for Developing Grounded Theory. CA: SAGE Publications, Thousand Oaks. http://doi.org/10.4135/9781452230153

[7] Alan Dix, Jennifer G. Sheridan, Stuart Reeves, Steve Benford, and Claire O'Malley. 2006. Formalising performative interaction. Lecture Notes in Computer Science (including subseries Lecture Notes in Artificial Intelligence and Lecture Notes in Bioinformatics) 3941 LNCS: 15-25. http://doi.org/10.1007/11752707_2

[8] Sebastian Draxler, Gunnar Stevens, Martin Stein, Alexander Boden, and David Randall. 2012. Supporting the Social Context of Technology Appropriation: On a Synthesis of Sharing Tools and Tool Knowledge. Human Factors in Computing Systems (CHI'12), ACM, 2835-2844. http://doi.org/10.1145/2207676.2208687

[9] Nicholas Epley and Juliana Schroeder. 2014. Mistakenly Seeking Solitude. Journal of Experimental Psychology 143, 5. http://doi.org/10.1037/a0037323

[10] Ava Fatah gen. Schieck, Vasillis Kostakos, and Alan Penn. 2009. Exploring Digital Encounters in the Public Arena. In Struppek M. Willis K., Roussos G., Chorianopoulos K. (ed.). Springer, London. http://doi.org/10.1007/978-1-84882-727-1_9

[11] Patrick Tobias Fischer and Eva Hornecker. 2012. Urban HCI : Spatial Aspects in the Design of Shared Encounters for Media Façades. Human Factors in Computing Systems (CHI '12), 307-316.

[12] Kenneth P. Fishkin, Thomas P. Moran, and Beverly L. Harrison. 1999. Embodied User Interfaces: Towards Invisible User Interfaces. Engineering for HumanComputer Interaction, 1997: 1-18. http://doi.org/10.1007/978-0-387-35349-4_1

[13] Dominic Furniss, Ann Blandford, and Paul Curzon. 2011. Confessions from a grounded theory PhD: experiences and lessons learnt. Human Factors in Computing Systems (CHI '11), ACM, 113-122. http://doi.org/10.1145/1978942.1978960

[14] Sarah Gallacher, Jenny O'Connor, Jon Bird, et al. 2015. Mood Squeezer : Lightening up the Workplace through Playful and Lightweight Interactions. Computer Supported Cooperative Work \& Social Computing (CSCW'15), ACM, 891-902. http://doi.org/10.1145/2675133.2675170

[15] William Gaver, John Bowers, Andy Boucher, Andy Law, Sarah Pennington, and Nicholas Villar. 2006. The history tablecloth: Illuminating domestic activity. Proceedings of the Conference on Designing Interactive Systems: Processes, Practices, Methods, and Techniques, DIS 2006: 199-208.

[16] Erving Goffman. 1963. Behavior in public places: notes on the social organization of gatherings. The Free Press, New York, NY, USA.

[17] Connie Golsteijn, Sarah Gallacher, Lisa Koeman, et al. 2015. VoxBox : a Tangible Machine that Gathers Opinions from the Public at Events. Proceedings of the 9th International Conference on Tangible, Embedded, and Embodied Interaction - TEI '15: 201-208. http://doi.org/10.1145/2677199.2680588

[18] Jens Emil Grønbæk. 2018. Designing for Interaction Proxemics : Configurations and Transitions. Conference on Human Factors in Computing Systems (CHI'18), 1-4. http://doi.org/10.1145/3170427.3173033

[19] Trine Heinemann. 2014. Breaching barriers to collaboration in public spaces. Tangible Embodied Interaction (TEI'14), 213-220. http://doi.org/10.1145/2540930.2540951

[20] Sara Heitlinger and Nick Bryan-Kinns. 2013. Understanding performative behaviour within contentrich Digital Live Art. Digital Creativity 24, 2: 111-118. http://doi.org/10.1080/14626268.2013.808962

[21] Eva Hornecker, Paul Marshall, and Yvonne Rogers. 2007. From entry to access - How shareability comes about. Proceedings of the 2007 Conference on Designing Pleasurable Products and Interfaces, DPPI'07, 328-342. http://doi.org/10.1145/1314161.1314191

[22] Steven Houben and Christian Weichel. 2013. Overcoming interaction blindness through curiosity objects. Extended Abstracts on Human Factors in Computing Systems (CHI EA '13), ACM, 1539-1544. http://doi.org/10.1145/2468356.2468631

[23] Lei Vincent Huang and Dan Wang. 2016. What a Surprise : Initial Connection with Coworkers on Facebook and Expectancy Violations. Computer Supported Cooperative Work (CSCW'16), 293-296.

[24] Giulio Jacucci, Peter Peltonen, Ann Morrison, Antti Salovaara, Esko Kurvinen, and Antti Oulasvirta. 2009. Ubiquitous media for collocated interaction. Computer Supported Cooperative Work (CSCW'09): 23-45. http://doi.org/10.1007/978-1-84882-727-1_2

[25] Clinton Jorge, Julian Hanna, Valentina Nisi, Nuno Nunes, Miguel Caldeira, and Giovanni Innella. 2013. Fostering ambiguity: Decontextualizing and Repurposing a Familiar Public Display. Biannual Conference of the Italian Chapter of SIGCHI (CHItaly '13), http://doi.org/10.1145/2499149.2499166

[26] Todd B. Kashdan and Michael F. Steger. 2007. Curiosity and pathways to well-being and meaning in 
life: Traits, states, and everyday behaviors. Motivation and Emotion 31, 3: 159-173. http://doi.org/10.1007/s11031-007-9068-7

[27] Sofie Kinch, E. Groenvall, M. Graves Petersen, and M. Kirkegaard Rasmussen. 2014. Encounters on a Shapechanging Bench Exploring atmospheres and social behaviour in situ. Tangible, Embedded and Embodied Interaction: $233-240$. http://doi.org/10.1145/2540930.2540947

[28] Eric Lesser and Larry Prusak. 1999. Communities of Practice, Social Capital and Organizational Knowledge. Information Systems Review 1, 1: 3-10. Retrieved from http://providersedge.com/docs/ km_articles/Cop__Social_Capital_-_Org_K.pdf

[29] Lian Loke, Toni Robertson, and Technology Sydney. 2013. Moving and Making Strange : An Embodied Approach to Movement-Based Interaction Design. TOCHI 20, 1. http://doi.org/10.1145/2442106.2442113

[30] David K. McGookin, Stephen A. Brewster, and Georgi Christov. 2014. Studying Digital Graffiti as a LocationBased Social Network. Human Factors in Computing Systems (CHI '14), ACM, 3269-3278. http://doi.org/10.1145/2556288.2557266

[31] Mary Ann McGrath and Cele Otnes. 1995. Unacquainted Influencers: When Strangers Interact in the Retail Setting. Journal of Business Research 32, 94: 261-272.http://doi.org/10.1016/0148-2963(94)00051-F

[32] Nemanja Memarovic, Sarah Clinch, and Florian Alt. 2015. Understanding Display Blindness in Future Display Deployments. Proceedings of the 4th International Symposium on Pervasive Displays (PerDis '15), ACM, 7-14. http://doi.org/10.1145/2757710.2757719

[33] Daniel Michelis and Jörg Müller. 2011. The Audience Funnel: Observations of Gesture Based Interaction With Multiple Large Displays in a City Center. International Journal of Human-Computer Interaction 27, 6: 562-579. http://doi.org/10.1080/10447318.2011. 555299

[34] Robb Mitchell and Thomas Olsson. 2017. Barriers for Bridging Interpersonal Gaps : Three Inspirational Design Patterns for Increasing Collocated Social Interaction. Proceedings of the 8th International Conference on Communities and Technologies (C\&T '17), ACM, 2-11.

[35] Beatrice Monastero and David McGookin. 2018. Traces: Studying a Public Reactive Floor-Projection of Walking Trajectories to Support Social Awareness. Human Factors in Computing Systems (CHI'18).

[36] Beatrice Monastero, David McGookin, and Giuseppe
Torre. 2016. Wandertroper: Supporting Aesthetic Engagement with Everyday Surroundings Through Soundscape Augmentation. Proceedings of the 15th International Conference on Mobile and Ubiquitous Multimedia: $129-140$. http://doi.org/10.1145/3012709.3012725

[37] Michael Muller, Katja Neureiter, Alina Krischkowsky, and Manfred Tscheligi. 2016. Collaborative Appropriation: How Couples, Teams, Groups and Communities Adapt and Adopt Technologies. Computer Supported Cooperative Work and Social Computing Companion (CSCW'16 Companion)., 473480. http://doi.org/10.1145/2818052.2855508

[38] Richard Phillips, Bethan Evans, and Stuart Muirhead. 2015. Curiosity, place and wellbeing: encouraging place-specific curiosity as a 'way to wellbeing.' Environment and Planning A 47, 11: 2339-2354. http://doi.org/10.1177/0308518X15599290

[39] Jason Potts and Julian Waters-lynch. 2016. The Social Economy of Coworking Spaces: A Focal Point Model of Coordination. 1-22. http://doi.org/10.13140/RG.2.1. 3150.3762

[40] Sara Price. 2008. A representation approach to conceptualizing tangible learning environments. Proceedings of the 2 nd international conference on Tangible and embedded interaction TEI 08: 151. http://doi.org/10.1145/1347390.1347425

[41] Sara Price, Taciana Pontual Falcão, Jennifer G. Sheridan, and George Roussos. 2009. The effect of representation location on interaction in a tangible learning environment. Proceedings of the 2 nd international conference on Tangible, Embedded and Embodied Interaction TEI 09: 85-92. http://doi.org/10.1145/1517664.1517689

[42] Pablo-Alejandro Quinones. 2014. Cultivating practice \& shepherding technology use: supporting appropriation among unanticipated users. Proceedings of the 17th ACM conference on Computer supported cooperative work \& social computing (CSCW'14), 305-318. http://doi.org/10.1145/2531602.2531698

[43] Jane Ritchie, Liz Spencer, A. Michael Huberman, and Matthew B. Miles. 2011. Qualitative Data Analysis for Applied Policy Research. In The Qualitative Researcher's Companion, A. Michael Huberman and Matthew B. Miles (eds.). SAGE Publications, 305-329. http://doi.org/10.4135/9781412986274.n12

[44] Yvonne Rogers, Jon Bird, Lisa Koeman, and Vaiva Kalnikait. 2014. What Chalk and Tape Can Tell Us : Lessons Learnt for Next Generation Urban Displays. Proceedings of The International Symposium on Pervasive Displays (PerDis '14), ACM, 130-135.

[45] Harvey Sacks. 1992. Lectures on Conversation. Blackwell Publishing. 
[46] Antti Salovaara, Antti Oulasvirta, and Giulio Jacucci. 2017. Evaluation of Prototypes and the Problem of Possible Futures. Human Factors in computing systems (CHI'17): 2064-2077. http://doi.org/10.1145/3025453. 3025658

[47] Ava Fatah gen. Schieck, Vassilis Kostakos, and Alan Penn. 2010. Exploring Digital Encounters in the Public Arena BT - Shared Encounters. In Katharine S Willis, George Roussos, Konstantinos Chorianopoulos and Mirjam Struppek (eds.). Springer London, London, 179-195. http://doi.org/10.1007/978-1-84882-727-1_9

[48] Jennifer G. Sheridan, Nick Bryan-Kinns, and Alice Bayliss. 2007. Encouraging witting participation and performance in Digital Live Art. People and Computers XXI HCI.But Not as We Know It - HCI 2007: The 21st British HCI Group Annual Conference 1. http://doi.org/10.14236/ewic/hci2007.2

[49] Agnis Stibe and Harri Oinas-kukkonen. 2014. Designing Persuasive Systems for User Engagement in Collaborative Interaction. European Conference on Information Systems (ECIS).

[50] Allan Stisen, Nervo Verdezoto, Henrik Blunck, Mikkel Baun Kjærgaard, and Kaj Grønbæk. 2016. Accounting for the Invisible Work of Hospital Orderlies : Designing for Local and Global Coordination. Computer supported cooperative work \& social computing CSCW'16: 980-992. http://doi.org/10.1145/2818048.2820006

[51] Alex S. Taylor, Siân Lindley, Tim Regan, and David Sweeney. 2015. Data-in-Place: Thinking through the Relations Between Data and Community. Proceedings of the 33rd Annual ACM Conference on Human Factors in Computing Systems (CHI '15), ACM, 28632872. http://doi.org/10.1145/2702123.2702558

[52] Sherry Turkle. 2011. Alone Together: Why We Expect More from Technology and Less from Each Other. Basic Books.
[53] Daniel Vogel and Ravin Balakrishnan. 2004. Interactive Public Ambient Displays: Transitioning from Implicit to Explicit, Public to Personal, Interaction with Multiple Users. Proceedings of the 17th annual ACM symposium on User interface software and technology (UIST '04), ACM, 137-146. http://doi.org/10.1145/1029632.1029656

[54] Etienne Wenger. 2001. Supporting communities of practice: a survey of community-oriented technologies. Homepage of Etienne Wenger Retrieved from httpwwwewengercomtech 2008, March: 1-3. Retrieved from http://www.ewenger.com/tech/

[55] Etienne Wenger. 2010. Communities of practice and social learning systems : the career of a concept. Social Learning Systems and Communities of Practice: 225 246. http://doi.org/doi: 10.1177/135050840072002

[56] William Hollingsworth Whyte. 2001. The Social Life of Small Urban Spaces. Project for Public Spaces.

[57] Julie R. Williamson and John Williamson. 2014. Analysing Pedestrian Traffic Around Public Displays. In Proceedings of The International Symposium on Pervasive Displays (PerDis '14), ACM, 13. http://doi.org/10.1145/2611009.2611022

[58] Niels Wouters, John Downs, Mitchell Harrop, et al. 2016. Uncovering the Honeypot Effect: How Audiences Engage with Public Interactive Systems. Proceedings of the 2016 ACM Conference on Designing Interactive Systems (DIS '16), ACM, 5-16. http://doi.org/10.1145/2901790.2901796

[59] Bengü Yücens and Ahmet Üzer. 2018. The relationship between internet addiction, social anxiety, impulsivity, self-esteem, and depression in a sample of Turkish undergraduate medical students. Psychiatry Research 267, June: 313-318. http://doi.org/10.1016/j.psychres.2018.06.033 